\title{
Guide de présentation pour les enquêtes
}

Correspondance : ccdr-rmtc@phac-aspc.gc.ca

Citation proposée : Un guide de présentation pour les enquêtes. Relevé des maladies transmissibles au Canada. 2017;43(9):208-10.

https://doi.org/10.14745/ccdr.v43i09a04f

Les enquêtes sont utiles pour décrire "ce qu'il en est ». On y a recours dans le domaine de la santé et de la recherche en santé publique pour en apprendre davantage sur les opinions, les connaissances et les pratiques actuelles, pour estimer la prévalence d'une maladie, pour analyser l'autoévaluation de l'état de santé, pour consigner les comportements à risque et ceux favorisant la santé, et pour recueillir des renseignements préliminaires en vue de futures études (1). Les méthodes d'enquête ont évolué, passant du formulaire papier à une diffusion faite surtout par des moyens électroniques. La plupart des enquêtes sont maintenant remplies individuellement en ligne, par courriel ou au moyen d'applications, ces trois possibilités pouvant aussi être combinées ( $p$. ex. un courriel $d$ 'invitation comprenant un hyperlien vers un questionnaire en ligne). Une revue Cochrane a révélé que les résultats des enquêtes menées sur les applications peuvent avoir des données équivalentes à celles obtenues par des méthodes plus traditionnelles, si le milieu, la fréquence et l'application clinique dans lesquels le questionnaire d'enquête a été validé restent les mêmes (2).

La recherche par enquêtes est utile pour la recherche exploratoire ou descriptive en raison de son coût relativement abordable, peut couvrir une vaste région géographique, englobe des milliers de personnes et assure une plus grande honnêteté lorsque l'anonymat est assuré. Les enquêtes ne sont pas utiles pour la recherche causale en raison du risque de facteurs de confusion et de biais (lorsque l'association observée entre deux variables est imputable à l'association des deux variables à une troisième variable non mesurée).

Habituellement, les enquêtes n'exigent pas d'examen éthique officiel. En revanche, le consentement éclairé est toujours indiqué et peut être respecté en précisant qui mène l'enquête, le but, la durée requise pour remplir l'enquête, les mesures incitatives et la politique de confidentialité des renseignements personnels. Pour les sondages en ligne, une pratique exemplaire consiste à calculer le taux de participation en mesurant le nombre de visiteurs uniques qui ont rempli la première page du sondage, divisé par le nombre de visiteurs uniques sur le site (3).

Dans la déclaration d'une recherche par enquêtes, il importe de décrire l'objectif, la population à l'étude, l'élaboration du questionnaire d'enquête et la façon dont l'étude a été menée, y compris la stratégie d'échantillonnage. Les résultats doivent inclure le taux de réponse, et la discussion doit tenir compte de la façon dont le taux de réponse, le biais de sélection, le biais lié aux réponses positives et les menaces à la fiabilité et à la validité des questions de l'enquête pourraient avoir influé sur les résultats.

Le Relevé des maladies transmissibles au Canada (RMTC) a dressé une liste de vérification comportant 22 éléments pour la déclaration des enquêtes en matière de maladies infectieuses, laquelle est basée sur la liste de vérification pour la déclaration des résultats d'enquêtes menées en ligne (Checklist for Reporting Results of Internet E-Surveys [CHERRIES]) (3), sur une liste de vérification précédente (4), ainsi que sur les pratiques exemplaires au chapitre des communications scientifiques (tableau 1). Une déclaration d'enquête compte généralement entre 1500 et 2000 mots. Comme pour toutes les soumissions, consultez le document Renseignements à l'intention des auteurs du RMTC pour les aspects généraux des exigences relatives à la préparation et à la soumission des manuscrits (5)

\section{Tableau 1 : Liste de vérification pour la déclaration des} enquêtes

\begin{tabular}{|c|c|c|}
\hline $\begin{array}{l}\text { Élément de la } \\
\text { déclaration }\end{array}$ & $N^{\circ}$ d'élément & Description \\
\hline \multicolumn{3}{|l|}{ Titre et résumé } \\
\hline Titre & 1 & $\begin{array}{l}\text { Donner un titre qui définit } \\
\text { le sujet de l'enquête et la } \\
\text { population à l'étude. }\end{array}$ \\
\hline Résumé & 2 & $\begin{array}{l}\text { Fournir un résumé structuré } \\
\text { de } 250 \text { mots qui comprend } \\
\text { l'objectif, la méthodologie } \\
\text { (y compris le milieu, la } \\
\text { population, ainsi que } \\
\text { l'élaboration et l'administration } \\
\text { du questionnaire de l'étude), } \\
\text { les résultats (y compris le taux } \\
\text { de réponse et les principales } \\
\text { conclusions) et la conclusion. }\end{array}$ \\
\hline \multicolumn{3}{|l|}{ Introduction } \\
\hline $\begin{array}{l}\text { Présentation de } \\
\text { I'enjeu }\end{array}$ & 3 & $\begin{array}{l}\text { Présenter le sujet de l'étude } \\
\text { et expliquer pourquoi il est } \\
\text { important. }\end{array}$ \\
\hline $\begin{array}{l}\text { Justification de } \\
\text { l'étude }\end{array}$ & 4 & $\begin{array}{l}\text { Citer les ouvrages pertinents et } \\
\text { déterminer dans quelle mesure } \\
\text { cette enquête apportera de } \\
\text { nouveaux éléments par rapport } \\
\text { à ceux que l'on connaît déjà. }\end{array}$ \\
\hline $\begin{array}{l}\text { Objectif et } \\
\text { justification }\end{array}$ & 5 & $\begin{array}{l}\text { Décrire clairement l'objectif de } \\
\text { l'étude et expliquer pourquoi } \\
\text { l'enquête était la méthode } \\
\text { appropriée pour y répondre. }\end{array}$ \\
\hline \multicolumn{3}{|l|}{ Méthodologie } \\
\hline $\begin{array}{l}\text { Population, } \\
\text { moment et lieu }\end{array}$ & 6 & $\begin{array}{l}\text { Décrire le milieu et la population } \\
\text { à l'étude, y compris les dates } \\
\text { pendant lesquelles l'enquête a } \\
\text { été menée. Indiquer s'il s'agissait } \\
\text { d'un échantillon de commodité. }\end{array}$ \\
\hline $\begin{array}{l}\text { Lien avec l'objectif } \\
\text { de la recherche }\end{array}$ & 7 & $\begin{array}{l}\text { Démontrer de quelle façon } \\
\text { les questions de l'enquête } \\
\text { répondent à l'objectif de la } \\
\text { recherche en indiquant les } \\
\text { différents sujets traités dans le } \\
\text { questionnaire. }\end{array}$ \\
\hline
\end{tabular}


Tableau 1 : Liste de vérification pour la déclaration des enquêtes (suite)

\begin{tabular}{|c|c|c|}
\hline $\begin{array}{l}\text { Élément de la } \\
\text { déclaration }\end{array}$ & $N^{\circ}$ d'élément & Description \\
\hline \multicolumn{3}{|c|}{ Méthodologie (suite) } \\
\hline $\begin{array}{l}\text { Élaboration du } \\
\text { questionnaire de } \\
\text { l'enquête }\end{array}$ & 8 & $\begin{array}{l}\text { Décrire la façon dont le } \\
\text { questionnaire d'enquête a } \\
\text { été élaboré, ce qui comprend } \\
\text { l'essai de la fiabilité et de la } \\
\text { validité, I'essai préliminaire et } \\
\text { l'essai pilote. }\end{array}$ \\
\hline $\begin{array}{l}\text { Technique } \\
\text { d'échantillonnage }\end{array}$ & 9 & $\begin{array}{l}\text { À moins que l'ensemble de } \\
\text { la population à l'étude ait } \\
\text { été interrogé, déterminer la } \\
\text { manière dont l'échantillonnage } \\
\text { a été effectué, y compris } \\
\text { les critères d'inclusion ou } \\
\text { d'exclusion (afin d'établir la } \\
\text { représentativité de l'échantillon) } \\
\text { et la façon dont l'enquête a été } \\
\text { envoyée (par courriel, Internet, } \\
\text { etc.). }\end{array}$ \\
\hline $\begin{array}{l}\text { Consentement } \\
\text { éclairé }\end{array}$ & 10 & $\begin{array}{l}\text { Décrire la façon dont les } \\
\text { participants potentiels ont été } \\
\text { informés au sujet des personnes } \\
\text { qui menaient l'enquête, du } \\
\text { but de l'enquête, de la durée } \\
\text { requise pour remplir l'enquête, } \\
\text { des mesures incitatives et de la } \\
\text { politique de confidentialité des } \\
\text { renseignements personnels. }\end{array}$ \\
\hline $\begin{array}{l}\text { Optimisation des } \\
\text { taux de réponse }\end{array}$ & 11 & $\begin{array}{l}\text { Préciser les procédures qui } \\
\text { ont été appliquées dans le but } \\
\text { d'optimiser le taux de réponse } \\
\text { (p. ex. si une lettre explicative } \\
\text { a été envoyée au préalable, ou } \\
\text { si des rappels ont été envoyés } \\
\text { aux non-répondants). }\end{array}$ \\
\hline Mesures & 12 & $\begin{array}{l}\text { Décrire toutes les mesures } \\
\text { utilisées dans le cadre de } \\
\text { l'étude, y compris la description } \\
\text { de la population à l'étude, } \\
\text { les mesures de résultats et } \\
\text { les facteurs de confusion } \\
\text { potentiels. }\end{array}$ \\
\hline Analyse & 13 & $\begin{array}{l}\text { Décrire la façon dont la taille de } \\
\text { l'échantillon a été calculée, ainsi } \\
\text { que toute analyse statistique } \\
\text { qui a été effectuée. }\end{array}$ \\
\hline \multicolumn{3}{|l|}{ Résultats } \\
\hline $\begin{array}{l}\text { Taux de } \\
\text { réponse et } \\
\text { représentativité } \\
\text { de l'échantillon }\end{array}$ & 14 & $\begin{array}{l}\text { Présenter le nombre de } \\
\text { réponses, le taux de réponse } \\
\text { et, dans la mesure du possible, } \\
\text { comparer les caractéristiques } \\
\text { de votre échantillon aux } \\
\text { renseignements disponibles au } \\
\text { sujet de la population à l'étude } \\
\text { (p. ex. une enquête menée } \\
\text { auprès des médecins pourrait } \\
\text { inclure l'âge, le sexe, les années } \\
\text { de pratique et l'emplacement). }\end{array}$ \\
\hline $\begin{array}{l}\text { Présentation des } \\
\text { résultats }\end{array}$ & 15 & $\begin{array}{l}\text { Présenter les résultats des } \\
\text { différents sujets dans l'ordre } \\
\text { correspondant aux sujets } \\
\text { décrits dans la méthodologie. }\end{array}$ \\
\hline
\end{tabular}

Tableau 1 : Liste de vérification pour la déclaration des enquêtes (suite)

\begin{tabular}{|c|c|c|}
\hline $\begin{array}{l}\text { Élément de la } \\
\text { déclaration }\end{array}$ & No d'élément & Description \\
\hline \multicolumn{3}{|l|}{ Résultats (suite) } \\
\hline $\begin{array}{l}\text { Tableaux et } \\
\text { figures }\end{array}$ & 16 & $\begin{array}{l}\text { Intégrer des tableaux et des } \\
\text { figures qui présentent les } \\
\text { principaux résultats, et veiller } \\
\text { à ce que tous les participants } \\
\text { soient représentés. }\end{array}$ \\
\hline \multicolumn{3}{|l|}{ Discussion } \\
\hline $\begin{array}{l}\text { Résumé des } \\
\text { principaux } \\
\text { résultats }\end{array}$ & 17 & $\begin{array}{l}\text { Résumer les principales } \\
\text { conclusions et indiquer la façon } \\
\text { dont elles répondent à l'objectif } \\
\text { de l'étude. Mettre en évidence } \\
\text { les résultats statistiquement } \\
\text { significatifs en matière de } \\
\text { pertinence clinique ou sociale. }\end{array}$ \\
\hline $\begin{array}{l}\text { Analyse } \\
\text { comparative }\end{array}$ & 18 & $\begin{array}{l}\text { Explorer la façon dont ces } \\
\text { résultats correspondaient ou } \\
\text { non avec les autres études } \\
\text { portant sur un sujet semblable } \\
\text { dans la littérature. }\end{array}$ \\
\hline $\begin{array}{l}\text { Forces et } \\
\text { faiblesses }\end{array}$ & 19 & $\begin{array}{l}\text { Cerner les forces et les } \\
\text { faiblesses de votre étude. } \\
\text { Prendre en compte les } \\
\text { répercussions qui surviendraient } \\
\text { si les répondants ne sont pas } \\
\text { représentatifs de l'ensemble de } \\
\text { l'échantillon, ou si l'échantillon } \\
\text { n'est pas représentatif de la } \\
\text { population concernée. Prendre } \\
\text { en considération la façon dont } \\
\text { un biais potentiel a été évité ou } \\
\text { non. }\end{array}$ \\
\hline Répercussions & 20 & $\begin{array}{l}\text { Tenir compte de la question } \\
\text { "qu'est-ce qui en résulte? » } \\
\text { dans vos constatations, c'est- } \\
\text { à-dire la façon dont elles } \\
\text { s'ajoutent aux connaissances } \\
\text { scientifiques, aux politiques ou à } \\
\text { la pratique. }\end{array}$ \\
\hline Prochaines étapes & 21 & $\begin{array}{l}\text { Proposer d'autres étapes ou } \\
\text { domaines de recherche à } \\
\text { explorer, sans trop dévier de vos } \\
\text { résultats. }\end{array}$ \\
\hline Conclusion & 22 & $\begin{array}{l}\text { Veiller à ce que la conclusion } \\
\text { intègre les principaux résultats } \\
\text { et qu'elle réponde à l'objectif de } \\
\text { l'enquête. }\end{array}$ \\
\hline
\end{tabular}

\section{Références}

1. Burns KE, Duffett M, Kho ME, Meade MO, Adhikari NK, Sinuff T, Cook DJ, for the ACCADEMY Group. A guide for the design and conduct of self-administered surveys of clinicians. CMAJ. 2008 Jul 29;179(3):245-52. DOI (http:// dx.doi.org/10.1503/cmaj.080372). PubMed (https://www. ncbi.nlm.nih.gov/entrez/query.fcgi?cmd=Retrieve\&db=PubM ed\&list_uids=18663204\&dopt=Abstract). 
2. Marcano Belisario JS, Jamsek J, Huckvale K, O'Donoghue J, Morrison CP, Car J. Comparison of self-administered survey questionnaire responses collected using mobile apps versus other methods. Cochrane Database Syst Rev. 2015 Jul 27;7:MR000042. PubMed (https://www.ncbi.nlm. nih.gov/entrez/query.fcgi?cmd=Retrieve $\& d b=$ PubMed\&li st_uids=26212714\&dopt=Abstract).

3. Eysenbach G. Improving the Quality of Web Surveys: The Checklist for Reporting Results of Internet E-Surveys (CHERRIES). J Med Internet Res. 2004 July-Sep; 6(3):e34. Correction J Med Internet Res 2012; 14(1):e8. DOI (http:// dx.doi.org/10.2196/jmir.6.3.e34) PubMed (https://www.ncbi.
nlm.nih.gov/entrez/query.fcgi?cmd=Retrieve\&db=PubMed\&l ist_uids=27355411\&dopt=Abstract).

4. Huston P. Reporting on surveys: information for authors and peer reviewers. CMAJ. 1996 Jun 1;154(11):1695-

704. PubMed (https://www.ncbi.nlm.nih.gov/ entrez/query.fcgi?cmd=Retrieve\&db=PubMed\&list_ uids $=8646657 \&$ dopt $=$ Abstract $)$.

5. Renseignements à l'intention des auteurs: janvier 2017. Relevé des maladies transmissibles au Canada. https://www. canada.ca/fr/sante-publique/services/rapports-publications/ releve-maladies-transmissibles-canada-rmtc/soumettrearticle-renseignements-a-intention-auteurs.html

\section{T A C \\ Conseil canadien de surveillance et d'accés aux traitements}

\section{La Carte de traitements (Carte'Tx) de CTAC}

est un outil en ligne qui permet aux membres de la communauté, aux fournisseurs de services et aux chercheurs du domaine des politiques d'obtenir de l'information sur les traitements contre le VIH et l'hépatite $\mathrm{C}$ qui sont couverts par les régimes publics d'assurance médicaments provinciaux, territoriaux et fédéraux.

Découvrez-la par vous mêmes à ctac.ca/cartetx où on y trouve plusieurs renseignements pertinents :

- critères cliniques d'admissibilité - coût moyen par dose

- aperçu des médicaments en

- disponibilité des médicaments cours d'élaboration

- accès entre les provinces et territoires

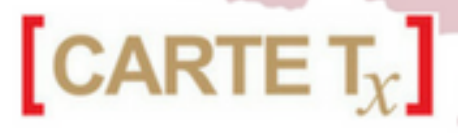

f/ctac.can | ctac.ca/cartetx | YouTube/ctactalks | info@ctac.ca | @ctac_can 